\title{
Differential role of CSF alpha-synuclein species, tau, and A $\beta 42$ in Parkinson's Disease
}

\section{Lucilla Parnetti ${ }^{1 *}$, Lucia Farotti ${ }^{1}$, Paolo Eusebi ${ }^{1,2}$, Davide Chiasserini ${ }^{3}$, Claudia De Carlo ${ }^{1}$, David Giannandrea ${ }^{1}$, Nicola Salvadori ${ }^{1}$, Viviana Lisetti ${ }^{1}$, Nicola Tambasco ${ }^{1}$, Aroldo Rossi ${ }^{1}$, Nour K. Majbour ${ }^{4}$, Omar El-Agnaf ${ }^{4,5}$ and Paolo Calabresi ${ }^{1,6}$}

${ }^{1}$ Clinica Neurologica, Università degli Studi di Perugia, Perugia, Italy

2 Dipartimento di Epidemiologia, Regione Umbria, Perugia, Italy

${ }^{3}$ Dipartimento di Scienze Farmaceutiche, Sezione di Biochimica, Università degli Studi di Perugia, Perugia, Italy

${ }^{4}$ Department of Biochemistry, Faculty of Medicine and Health Sciences, United Arab Emirates University, Al Ain, United Arab Emirates

${ }^{5}$ Faculty of Medicine, King Abdulaziz University, Jeddah, Saudi Arabia

${ }^{6}$ IRCCS Fondazione S. Lucia, Roma, Italy

\section{Edited by:}

Manuel Menéndez-González, Hospital Álvarez-Buylla, Spain

\section{Reviewed by:}

Jose-Luis Gonzalez De Aguilar, University of Strasbourg, France Pooja Rao, European Neuroscience Institute, Germany

Victoria M. Leavitt, Manhattan

Memory Center, USA

*Correspondence:

Lucilla Parnetti, Clinica Neurologica, Università di Perugia, Sant'Andrea delle Fratte, 06132 Perugia, Italy e-mail:parnetti@unipg.it
There is a great interest in developing cerebrospinal fluid (CSF) biomarkers for diagnosis and prognosis of Parkinson's disease (PD). CSF alpha synuclein ( $\alpha$-syn) species, namely total and oligomeric $\alpha$-syn (t- $\alpha$-syn and o- $\alpha$-syn), have shown to be of help for PD diagnosis. Preliminary evidences show that the combination of CSF t- $\alpha$-syn and classical Alzheimer's disease $(A D)$ biomarkers- $\beta$-amyloid 1-42 $\left(A \beta_{42}\right)$, total tau (t-tau), phosphorylated tau ( $p$-tau) - differentiate PD patients from controls, and that reduced levels of $A \beta_{42}$ represent a predictive factor for development of cognitive deterioration in PD. In this prospective study carried out in 44 PD patients and 25 neurological controls we wanted to verify whether the combination of CSF $\alpha$-synuclein species-t- $\alpha$-syn and o- $\alpha$-syn-and classical $A D$ biomarkers may help in differentiating PD from neurological controls, and if these biomarkers may predict cognitive decline. The median of follow-up duration was 3 years (range: 2-6 years). Mini Mental State Examination (MMSE) and Montreal Cognitive Assessment (MoCA) were used for monitoring cognitive changes along time, being administered once a year. Oligo/total $\alpha$-syn ratio (o/t- $\alpha$-syn ratio) confirmed its diagnostic value, significantly contributing to the discrimination of PD from neurological controls. A greater diagnostic accuracy was reached when combining o/t- $\alpha$-syn and $A \beta_{42} /$ tau ratios (Sens $=0.70, \mathrm{Spec}=0.84, \mathrm{AUC}=0.82 ; \mathrm{PPV}=0.89, \mathrm{NPV}=0.62, \mathrm{LR}+=4.40, \mathrm{DOR}=$ 12.52). Low CSF $A \beta_{42}$ level was associated with a higher rate of MMSE and MoCA decline, confirming its role as independent predictive factor for cognitive decline in PD. None of the other biomarkers assessed (t-tau, p-tau, t- $\alpha$-syn and o- $\alpha$-syn) showed to have prognostic value. We conclude that combination of CSF o/t- $\alpha$-syn and $A \beta_{42} /$ tau ratios improve the diagnostic accuracy of PD. PD patients showing low CSF $A \beta_{42}$ levels at baseline are more prone to develop cognitive decline.

Keywords: cerebrospinal fluid biomarkers, alpha synuclein, total tau, phosphorylated tau, $A \beta_{42}$, Parkinson's disease, cognitive decline

\section{INTRODUCTION}

Parkinson's disease $(\mathrm{PD})$ is a common neurodegenerative disorder evolving, in a substantial proportion of patients, to dementia. Although it is defined as a typical movement disorder and its diagnosis is mainly based on motor-related clinical criteria, other functional domains are also involved. Accordingly, post-mortem findings of alpha-synuclein ( $\alpha$-syn) pathology - the histopathologic hallmark of PD—show that the involvement of the dorsal motor nucleus of the vagal nerve and the olfactory bulb takes place much earlier before midbrain involvement (Braak et al., 2003).

$\mathrm{PD}$ is a complex neurodegenerative disorder in which many different pathophysiological processes take place, such as protein aggregation, oxidative damage and lysosomal dysfunction (Parnetti et al., 2013). Concomitant pathologies (i.e., Alzheimer and Lewy bodies pathologies) resulting from the mutual interaction between $\mathrm{A} \beta_{42}$, tau and $\alpha$-syn during the course of the disease have major role in the neuropathological processes underlying dementia in PD (Tsigelny et al., 2008; Ciaccioli et al., 2013). In PD the spread of fibrillar $\alpha$-syn pathology from the brainstem to limbic and neocortical structures, and the cortical deposition of $\beta$-amyloid plaques, represent major events (Compta et al., 2011; Irwin et al., 2012). Co-occurrence of tau and $\alpha$-syn pathology has been found in neurons of brains affected by tauopathies and synucleinopathies, including PD (Vekrellis et al., 2011). Also, $\alpha$-syn causes aggregation and polymerization of tau, which then induces the formation of intracellular amyloid-tau inclusions (Waxman and Giasson, 2011).

Since molecular changes in the brain are reflected in cerebrospinal fluid (CSF) composition, the CSF represents an ideal 
source for biomarkers of different pathophysiological processes characterizing the early phases of the disease, when the clinical diagnosis is more challenging. For example, $A \beta_{42}$, total tau ( $t$-tau) and phosphorylated tau (p-tau) are state markers of Alzheimer's disease $(A D)$, as they reliably reflect $A D$ pathology also in predementia phases. This knowledge has been translated into operational diagnostic criteria (Dubois et al., 2010). Analogously, there is great interest for improving early diagnosis in $\mathrm{PD}$, hopefully in the pre-motor phases, as well as for detecting PD patients at risk of dementia.

Currently, detection of reliable CSF biomarkers for PD is under intensive investigation. Several recent studies have explored the potential use of CSF total $\alpha$-syn ( $\mathrm{t}-\alpha$-syn) as a putative PD biomarker. A clear trend of lower CSF t- $\alpha$-syn levels in PD and other synucleinopathies has been consistently reported (Tokuda et al., 2006; Noguchi-Shinohara et al., 2009; Spies et al., 2009; Hong et al., 2010; Mollenhauer et al., 2011; Parnetti et al., 2011; Aerts et al., 2012; Tateno et al., 2012), although with a large overlap between the PD and control groups (Noguchi-Shinohara et al., 2009; Spies et al., 2011). Therefore, the measurement of CSF t- $\alpha$-syn doesn't seem to have enough specificity to correctly discriminate patients with synucleinopathies from normal individuals or other neurodegenerative diseases. The measurement in CSF of other $\alpha$-syn species, namely soluble oligomers (o- $\alpha$ syn) has improved the discrimination between PD and other diseases. O- $\alpha$-syn levels are elevated in brain homogenates in PD and dementia with Lewy bodies compared with normal brains (Paleologou et al., 2009) suggesting a role for $o-\alpha$-syn in PD pathogenesis. CSF o- $\alpha$-syn levels and $\mathrm{o} / \mathrm{t}-\alpha$-syn ratio have been consistently found to be significantly higher in PD patients as compared to other neurological disorders, with good sensitivity and specificity, as confirmed in independent reports (Tokuda et al., 2010; Park et al., 2011; Sierks et al., 2011; Parnetti et al., 2014). The high risk of cognitive impairment in PD also calls for biomarkers able to predict dementia onset. Many studies have focused on classical AD CSF biomarkers (Parnetti et al., 2008; Compta et al., 2009; Alves et al., 2010; Montine et al., 2010; Siderowf et al., 2010; Leverenz et al., 2011) and most of them have identified the reduction of $\mathrm{CSF} A \beta_{42}$ levels as a prognostic factor for cognitive impairment in PD. Data on the possible relationship between $\alpha$-syn species and the risk of dementia in PD are still scanty.

In this study we evaluated both the diagnostic accuracy and the capability in predicting cognitive decline of CSF AD biomarkers ( $A \beta_{42}$, t-tau, $\mathrm{p}$-tau and $\mathrm{A} \beta_{42} / \mathrm{t}$-tau ratio) and $\alpha$-syn species ( $\mathrm{t}-\alpha-$ syn, o- $\alpha$-syn and o/t- $\alpha$-syn ratio) in PD patients and neurological controls with a median follow up duration of 3 years.

\section{MATERIALS AND METHODS PATIENTS}

The subjects included in this study (44 PD, 25 neurological controls) were consecutively recruited between 2007 and 2011 and followed-up. They underwent a baseline clinical examination by experienced neurologists, detailed neuropsychological testing, blood chemistry, neuroimaging (computed tomography and/or magnetic resonance imaging), and lumbar puncture. CSF was collected according to the hospital standard protocol and with the local ethical committee approval, after informed written consent was given by the patient. All PD patients fulfilled the United Kingdom Parkinson's Disease Society Brain Bank clinical diagnostic criteria. All of them were treated with L-DOPA and the great majority (39 out of 44 ) were also taking DA-agonists. PD patients had good control of motor symptoms (mean UPRDS-III $25.39 \pm 12.97)$, and were functionally independent or minimally dependent (Hoehn and Yahr, 1-2.5). Disease duration was calculated from the onset of the first motor symptoms to the time of lumbar puncture. As a control group, 25 cognitively normal age-matched subjects who underwent lumbar puncture as a part of diagnostic work up for other neurological conditions (OND) were recruited to our study. The OND group included: primary headache $(n=15)$, postural instability $(n=3)$, seizures $(n=2)$ and polyneuropathy $(n=5)$. Follow-up visits included clinical examination and neuropsychological testing carried out yearly by means of MMSE (Folstein et al., 1975), a popular screening tool mostly measuring cortical functions with special attention to memory, and MoCA (Gill et al., 2008), another screening tool assessing executive functions.

\section{CSF SAMPLING}

Lumbar puncture was performed between 8:00 and 10:00 A.M., after an overnight fast. CSF $(10 \mathrm{~mL})$ was collected in sterile polypropylene tubes, centrifuged for $10 \mathrm{~min}$ at $2000 \times \mathrm{g}$, and $0.5-\mathrm{mL}$ aliquots were immediately frozen at $-80^{\circ} \mathrm{C}$. None of the samples was contaminated by blood during the procedure (samples showing an erythrocyte count $>500 / \mathrm{mm}^{3}$ were not included in the study). CSF $A \beta_{42}$, t-tau and p-tau were measured with ELISA assays (Innotest $\beta$ Amyloid 1-42, hTAU-Ag, p-TAU 181 Ag; Innogenetics NV, Gent, Belgium, now Fujirebio). For CSF $\alpha$-syn, $0.5 \mathrm{~mL}$ samples were thawed on ice and then divided into aliquots of $110 \mu \mathrm{L}$ in siliconized tubes containing a cocktail of protease inhibitors, including AEBSF, aprotinin, E-64, EDTA, and leupeptin (Calbiochem-Novabiochem Corporation, San Diego, CA), and $0.05 \%$ Tween 20, and the samples were then stored at $-80^{\circ} \mathrm{C}$ until used in the immunoassay for $\alpha$-syn. While CSF $\mathrm{t}-\alpha$-syn is expressed as $\mathrm{ng} / \mathrm{mL}, \mathrm{CSF} \mathrm{o} / \mathrm{t}-\alpha$-syn ratio is expressed in CPS (counts per second). All the samples were obtained at the Section of Neurology, Perugia General Hospital, according to the protocol approved by the Regional Ethical Committee (Prot. N. 19369/AV), after informed written consent was obtained.

\section{IMMUNOASSAY FOR $\alpha$-SYNUCLEIN IN CSF}

Total and oligomeric CSF $\alpha$-syn were measured as previously reported (Tokuda et al., 2006). Briefly, for CSF $\mathrm{t}-\alpha-$ syn anti-human $\alpha$-syn monoclonal antibody (clone Syn211) (Santa Cruz Biotechnology, USA) was used for capturing while the anti-human $\alpha$-syn polyclonal antibody FL-140 (Santa Cruz Biotechnology, USA) was used for antigen detection. The standard curve for the ELISA assay was constructed using recombinant human $\alpha$-syn solution at different concentrations diluted in blocking buffer. For $\alpha$-syn oligomers, the antibody clone Syn211 was used for capturing, while biotinylated Syn211 (Santa Cruz Biotechnology, USA) was used for antigen detection. The plate was incubated with $50 \mu \mathrm{L} /$ well of ExtrAvidin-Peroxidase 
(Sigma-Aldrich, UK) and with the enhanced chemiluminescent substrate. For both immunoassays, the samples were screened in blind fashion and were randomly tested. A series of internal controls were run to check for run-to-run variations.

\section{DATA ANALYSIS}

Statistical analyses were performed using $\mathrm{R}$ software v. 2.15 ( $\mathrm{R}$ Core Team, 2013). Continuous variables were described by median and ranges since data distributions were skewed. Correlations were calculated using Spearman's Rho (r). KruskalWallis test was initially used for comparisons between the two diagnostic groups $(p<0.05)$. The accuracy of the diagnostic value of the biomarkers was assessed by area under the curve (AUC) of the receiver operating characteristic (ROC) curve (Robin et al., 2011; Eusebi, 2013). Cut-off values were calculated using sensitivity and specificity values that maximized Youden's index (sensitivity + specificity -1 ). For evaluating the role of multiple biomarkers a multivariable logistic regression approach was used. With the aim to find the best predictors of PD to be included in the final model, we considered all the CSF enzyme activities which had already shown significant differences between OND and PD groups after the univariate analysis.

Multivariate linear regression analysis was used for analyzing the biomarker role in predicting cognitive decline. Change in MMSE and MoCA score were considered as dependent variables. Multiple imputations for missing values were performed in multivariable analyses (Rubin, 1987). Missing data were filled in five times to generate five complete data sets. The completed datasets were analyzed by using the mixed-effects model and the results were combined for the inference.

\section{RESULTS}

\section{DESCRIPTIVE ANALYSIS}

Demographic data, clinical features, and biomarkers values are listed in Table 1. As expected, no significant difference between $\mathrm{PD}$ and OND groups was found with respect to age, gender, and follow-up duration. Both MMSE and MoCA scores were significantly lower at baseline $(p=0.009$ and $p=0.025$, respectively) and after follow-up $(p=0.004$ and $p<0.001$, respectively). Follow-up observations refer to the last visit carried out.

\section{CSF BIOMARKERS IN DIAGNOSTIC GROUPS}

Values of CSF biomarkers showed substantial overlap between the two groups (Table 2 and Figure 1). Although the differences did not reach the statistical significance, in PD group median $\mathrm{A} \beta_{42}$ levels were higher as opposite to lower median t-tau levels. As a consequence, $A \beta_{42} / t$-tau ratio was significantly increased in the PD group with respect to OND subjects $(p<0.01$, Table 2). Analogously to previous observation (Balducci et al., 2007), a significant decrease of $\mathrm{t}-\alpha$-syn $(p=0.015)$ and an increase of $o$ - $\alpha$-syn levels $(p=0.041)$ were found in PD group (Table 2). Interestingly the o/t- $\alpha$-syn ratio greatly improved the discrimination between PD and OND groups $(p<0.001$, Table 2). ROC analysis showed a sensitivity of 0.82 and a specificity of 0.56 for $A \beta_{42} / t$-tau ratio. T- $\alpha$-syn had a sensitivity of 0.59 and a specificity of 0.80 . O- $\alpha$-syn disclosed a sensitivity of 0.89 and a specificity of 0.48 . $\mathrm{O} / \mathrm{t}-\alpha$-syn ratio reached the best diagnostic performance having a sensitivity of 0.82 and a specificity of 0.64 .

In Table 3 the correlation analysis for all the CSF biomarkers considered is reported. Interestingly, in the PD group, an inverse association between $t-\alpha$-syn and $t$-tau was found. Such a negative correlation was also observed in the OND group, where it did not reach the statistical significance. As expected, in the OND group a significant positive association between $t-\alpha$-syn and $A \beta_{42} /$ tau ratio was observed.

Table 4 reports the correlation analysis between CSF biomarkers and clinical parameters in $\mathrm{OND}$ and $\mathrm{PD}$ groups. In $\mathrm{PD}$ t-tau was positively correlated with the H\&Y stage, as opposite to the $A \beta_{42} / t$-tau ratio, which was inversely related to $H \& Y$. Cognitive changes along time were measured as points lost in MMSE and MoCA scores between baseline and follow-up visits. In $\mathrm{PD} \mathrm{A} \beta_{42}$ was negatively correlated with decline in MMSE and MoCA scores. $A \beta_{42} / t$-tau ratio was negatively correlated with decrease in MMSE score. In OND group no significant correlation was found between CSF parameters and decrease in MMSE and MoCA scores.

\section{MULTIPLE BIOMARKERS EVALUATION IN DIAGNOSTIC GROUPS}

In order to assess the diagnostic performance of multiple biomarkers combination a logistic regression approach was used. Table 5 shows a summary of the best model according to several measures of test effectiveness, including sensitivity and specificity, positive and negative predictive values, positive/negative likelihood ratio, AUC and diagnostic odds ratio (DOR). The model included $\mathrm{A} \beta_{42} / \mathrm{t}$-tau and $\mathrm{o} / \mathrm{t}-\alpha$-syn ratios, which together reached a specificity of $84 \%$ and a sensitivity of $70 \%$.

Figure 2 shows how the model separates PD patients from OND, allowing for a good discrimination of PD and how the model predictions reach a superior diagnostic performance with respect to the o/t- $\alpha$-syn or $A \beta_{42} / t$-tau ratios, separately.

\section{CSF BIOMARKERS FOR PREDICTING COGNITIVE DECLINE IN PD}

As reported in the previous section, $A \beta_{42}$ and the $A \beta_{42} / t$-tau ratio were the only parameters showing a correlation with MMSE and MoCA scores. To investigate the relationship between the decrease of these two neuropsychological measurements with $\mathrm{A} \beta_{42}$ and $\mathrm{A} \beta_{42} / \mathrm{t}$-tau ratio, a multivariate linear regression model was applied, adjusting for the baseline values and follow-up duration (Table 6). MMSE score decrease confirmed to be significantly associated with low CSF $A \beta_{42}$ levels at baseline; the same trend was also observed for MoCA scores; $A \beta_{42} / t$-tau ratio was not significantly associated with cognitive decline.

\section{DISCUSSION}

As in other neurodegenerative disorders, PD is characterized by a large time gap between the beginning of neurodegenerative processes and the onset of clinical neurological manifestations. The disease's natural history includes a first asymptomatic stage, followed by a long pre-motor phase; finally, when the classical motor symptoms appear, the majority of nigral dopaminergic neurons are already affected by degeneration. The classical diagnostic criteria for PD mostly rely on motor symptoms, making the formulation of an early diagnosis very challenging. Another challenge 
Table 1 | Demographic data and clinical features for OND and PD.

\begin{tabular}{|c|c|c|c|}
\hline & OND & PD & $p$-value \\
\hline$N$ & 25 & 44 & - \\
\hline Age & 58 (R 31-78; IQR 47-73) & 66 (R 41-79; IQR 57.8-72) & 0.117 \\
\hline PD duration (years) & - & 3 (R 1-9; IOR 1-5.25) & - \\
\hline Hoehn and Yahr score & - & 2 (R 1-4; IQR 1.5-2.5) & - \\
\hline MoCA score at baseline & 28 (R 27-30; IOR 25.5-28.5) & 25.5 (R 17-30; IQR 22.8-28) & 0.025 \\
\hline MoCA score at follow-up & 26 (R 20-29; IQR 24-27) & 22 (R 10-28; IQR 18.75-25) & $<0.001$ \\
\hline Follow-up duration (years) & 4 (R 2-7; IQR 3-5) & 2 (R 1-7; IQR 2-6) & 0.197 \\
\hline
\end{tabular}

$P$-values, count, and percentages for sex and medians, ranges (R), interquartile ranges (IQR) for the other variables.

Table 2 | CSF biomarkers in PD and OND.

\begin{tabular}{|c|c|c|c|c|c|c|c|}
\hline & OND & PD & $p$-value & AUC & Sens & Spec & cut-off \\
\hline$A \beta_{42}$ & 530 (431-752) & 693 (493-852) & 0.057 & $0.64(0.51-0.78)$ & 0.59 & 0.72 & 636.00 \\
\hline t-tau & 194 (117-257) & 146 (109-204) & 0.085 & $0.63(0.48-0.77)$ & 0.64 & 0.68 & 159.00 \\
\hline p-tau & $19(11-24)$ & $19.5(9.75-30.25)$ & 0.793 & $0.52(0.38-0.66)$ & 0.36 & 0.80 & 25.50 \\
\hline $\mathrm{A} \beta_{42} /$ t-tau ratio & $2.85(1.88-4.88)$ & $4.70(3.47-6.38)$ & 0.004 & $0.71(0.59-0.84)$ & 0.82 & 0.56 & 3.15 \\
\hline t- $\alpha-s y n$ & $36.5(25.8-49.6)$ & $22.15(11.86-38.64)$ & 0.015 & $0.68(0.55-0.81)$ & 0.59 & 0.80 & 24.45 \\
\hline o- $\alpha$-syn & 3139 (1500-6140) & 4838 (3049-8141) & 0.041 & $0.72(0.59-0.84)$ & 0.89 & 0.48 & 2565.50 \\
\hline o/t- $\alpha$-syn ratio & $0.021(0.014-0.043)$ & $0.061(0.034-0.175)$ & $<0.001$ & $0.78(0.67-0.89)$ & 0.82 & 0.64 & 0.03 \\
\hline
\end{tabular}

Median values with interquartile ranges (IQR); ROC analysis summary with AUC (95\% CI), sensitivity and specificity.

for the research focused on this disorder is the understanding of the mechanisms underlying the development of dementia taking place in a subgroup of parkinsonian patients. It would be very important to have the possibility to individuate those patients at risk to develop this devastating complication to initiate possible protective pharmacological and non-pharmacological interventions. Thus, the availability of objective measures such as reliable "biomarkers," indicators of biological/pathogenetic processes, will be of great importance both for diagnostic accuracy and prognostic evaluation.

In this context, CSF analysis might be of great importance since CSF dynamically reflects the pathophysiological processes taking place in the brain. At present, CSF biomarkers are a routine analysis for early diagnosis of AD. Accordingly, increasing interest is focused on CSF biomarkers in PD, with major expectations on $\alpha$-syn species and other misfolding proteins, namely $\beta$-amyloid and tau. With respect to diagnostic performance, data available so far indicate that there is not a unique ideal CSF biomarker, rather the combination of molecules related to different pathophysiological pathways involved in $\mathrm{PD}$ may represents a good strategy for obtaining a more accurate diagnosis (Parnetti et al., 2014). Concerning the prediction of cognitive decline in $\mathrm{PD}$, the most consistent role as predictive factor is played by low CSF $\mathrm{A} \beta_{42}$ levels (Parnetti et al., 2008; Alves et al., 2010; Siderowf et al., 2010; Leverenz et al., 2011) although also tau species have been postulated to represent prognostic factors (Zhang et al., 2013). Interestingly, a recent investigation (Kang et al., 2013) carried out in drug-naïve patients with early $\mathrm{PD}$, showed slightly lower CSF levels of both t-tau and $t-\alpha$-syn in PD compared to healthy controls. This finding offered the Authors the opportunity to speculate that the interaction between tau proteins and $\alpha$-syn may limit the release of tau proteins into CSF.

In this investigation we assessed both the diagnostic accuracy and the performance in predicting cognitive decline of the combination of CSF AD biomarkers $\left(\mathrm{A} \beta_{42}\right.$, t-tau, p-tau, and $\mathrm{A} \beta_{42} / \mathrm{t}$-tau ratio) and $\alpha$-syn species ( $\mathrm{t}-\alpha$-syn, $o-\alpha$-syn, and $\mathrm{o} / \mathrm{t}-\alpha$-syn ratio) in a cohort of PD patients and neurological controls followed up for 2-6 years (median follow-up duration: 3 years).

With respect to the diagnostic performance of the biomarkers considered, none of them demonstrated acceptable values in terms of sensitivity and specificity when taken separately. $A \beta_{42} / t-$ tau and $\mathrm{o} / \mathrm{t}-\alpha$-syn ratios showed good sensitivity $(0.82)$ but low specificity ( 0.56 and 0.64 , respectively). While the usefulness of $\mathrm{o} / \mathrm{t}-\alpha$-syn ratio in discriminating $\mathrm{PD}$ and controls has already been reported in recent investigations (Tokuda et al., 2010; Park et al., 2011; Sierks et al., 2011; Parnetti et al., 2014), the $A \beta_{42} / t$ tau ratio deserves some comments. Interestingly, in the PD group, we found slightly higher values of $A \beta_{42}$ together with lower values of $\mathrm{t}$-tau as compared to OND group. As a consequence, the mean value of $A \beta_{42} / t$-tau ratio was significantly higher in $P D$ patients with respect to the OND group. This may be due to the fact that our control group was not including healthy subjects, being composed by patients with other neurological diseases. Interestingly, reduced CSF $A \beta_{42}$ levels at baseline represented a 

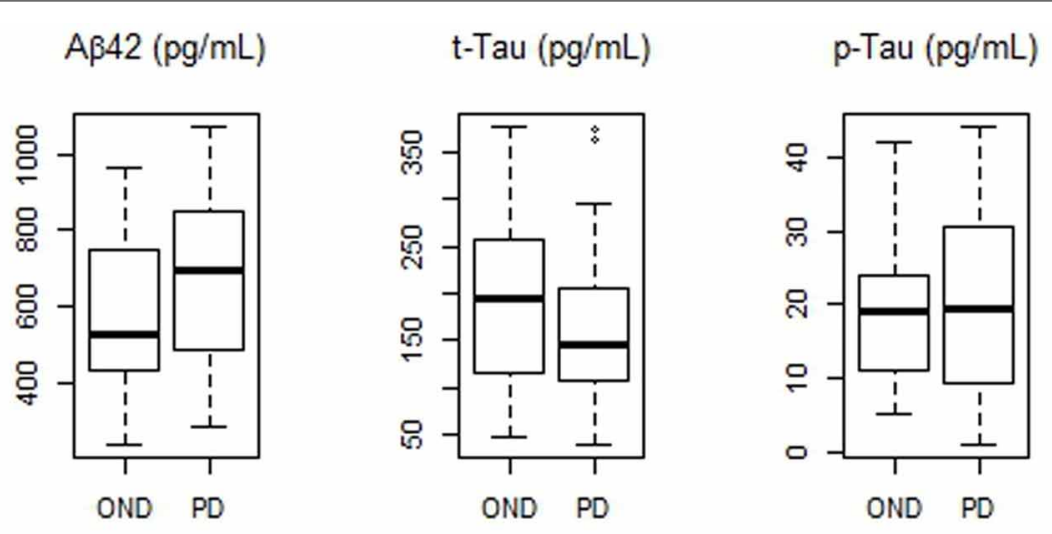

\section{A $342 /$ t-tau ratio}

t- $\alpha$-syn $(\mathrm{ng} / \mathrm{mL})$

0- $\alpha-\operatorname{syn}(C P S)$

\section{o/t-o-syn ratio}
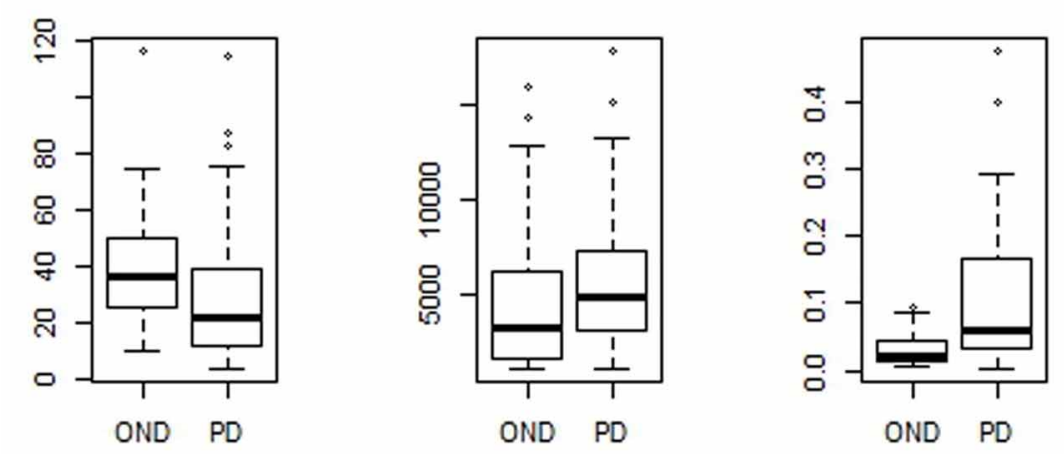

FIGURE 1 | Boxplots of CSF biomarkers values observed in PD and OND cohorts. The horizontal bold lines indicate the medians; the lower part of boxes indicates the first quartile and the upper part the third quartile; the dashed vertical lines indicate the range of values.

Table 3 | Spearman's rank correlation matrix for CSF biomarkers in PD and OND groups.

\begin{tabular}{|c|c|c|c|c|c|c|c|c|}
\hline & & $A \beta_{42}$ & t-tau & p-tau & $A \beta_{42} /$ tau ratio & t-asyn & o-asyn & o/t-asyn ratio \\
\hline \multirow[t]{4}{*}{ PD } & $A \beta_{42}$ & 1.00 & & & & & & \\
\hline & t-tau & 0.07 & 1.00 & & & & & \\
\hline & $\mathrm{A} \beta_{42} /$ t-tau ratio & - & - & $-0.44^{* *}$ & 1.00 & & & \\
\hline & t- $\alpha$-syn & -0.25 & $-0.33^{*}$ & -0.25 & 0.13 & 1.00 & & \\
\hline \multirow[t]{5}{*}{ OND } & $A \beta_{42}$ & 1.00 & & & & & & \\
\hline & t-tau & 0.28 & 1.00 & & & & & \\
\hline & p-tau & 0.11 & $0.56^{* *}$ & 1.00 & & & & \\
\hline & $\mathrm{A} \beta_{42} /$ t-tau ratio & - & - & $-0.41 *$ & 1.00 & & & \\
\hline & $\mathrm{t}-\alpha-\operatorname{syn}$ & 0.24 & -0.25 & -0.24 & $0.43^{*}$ & 1.00 & & \\
\hline
\end{tabular}

${ }^{*} p<0.05,{ }^{* *} p<0.01,{ }^{* * *} p<0.001$.

predictive factor for cognitive decline only in the PD group. In fact, only in PD patients lower CSF A $\beta_{42}$ levels were correlated to a more marked decrease in MMSE and MoCA scores at followup. The finding of reduced CSF $A \beta_{42}$ levels in PD patients is quite controversial, being reported in some (Sjögren et al., 2002; Zhang et al., 2008; Alves et al., 2010) but not in other papers (Pøikrylová Vranová, 2010; Siderowf et al., 2010; Leverenz et al., 2011). 
Table 4 | Spearman's rank correlations between CSF biomarkers, age, disease duration, and clinical scores in PD group.

\begin{tabular}{|c|c|c|c|c|c|c|}
\hline & & Age & Disease duration & Hoehn and Yahr scale & MMSE score decrease & MoCA score decrease \\
\hline & t-tau & $0.31 *$ & 0.04 & $0.39 * *$ & 0.18 & 0.08 \\
\hline & $\mathrm{A} \beta_{42} /$ t-tau ratio & $-0.42^{* *}$ & -0.11 & $-0.50^{* * *}$ & $-0.37^{*}$ & -0.26 \\
\hline & t- $\alpha$-syn & 0.00 & 0.30 & 0.10 & 0.06 & -0.03 \\
\hline \multirow[t]{5}{*}{ OND } & $A \beta_{42}$ & -0.37 & & & -0.01 & -0.38 \\
\hline & t-tau & 0.18 & & & 0.17 & 0.09 \\
\hline & p-tau & 0.18 & & & -0.26 & 0.26 \\
\hline & $A \beta_{42} /$ t-tau ratio & $-0.43^{*}$ & & & -0.12 & -0.24 \\
\hline & t- $\alpha$-syn & $-0.41^{*}$ & & & -0.37 & -0.07 \\
\hline
\end{tabular}

${ }^{*} p<0.05,{ }^{* *} p<0.01,{ }^{* * *} p<0.001$

Table 5 | Logistic regression analysis of multiple CSF biomarkers between PD and OND.

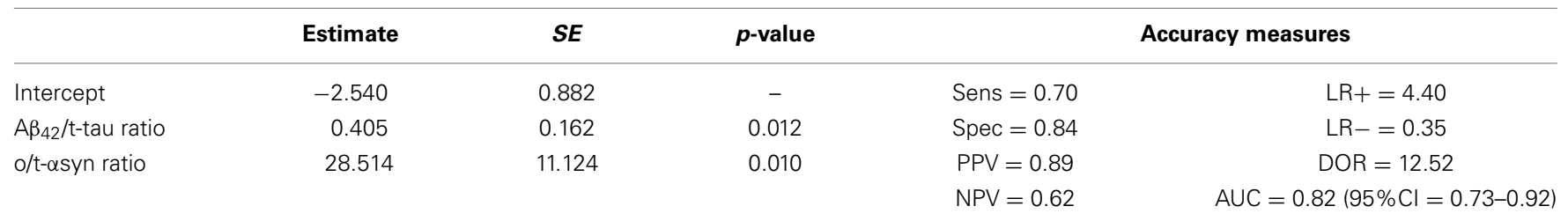

A

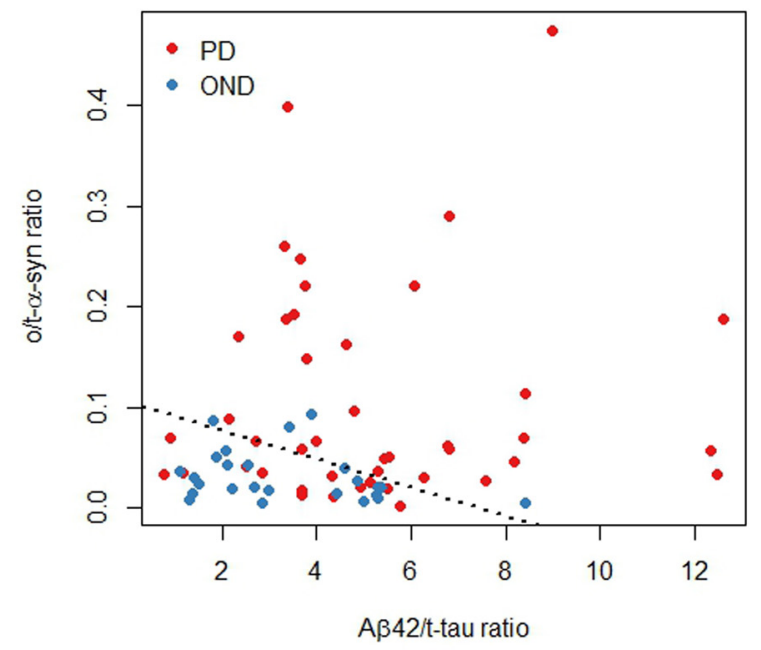

FIGURE 2 | $A \beta_{42} / t$-tau and $o / t-\alpha$-syn ratios in PD and OND.

(A) Scatterplot: the dashed line represents a partition of the o/t- $\alpha$-syn and $A \beta_{42} / t$-tau space such that below the line the model predicts OND,

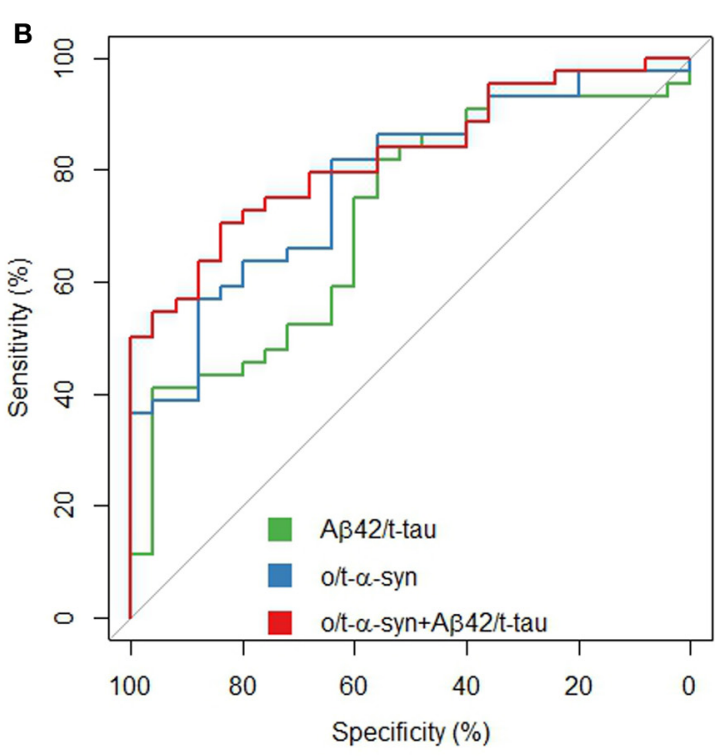

above the line the model predicts PD (B) ROC curves of $A \beta_{42} / t$-tau and $\mathrm{o} / \mathrm{t}-\alpha$ syn ratios and the fitted values of the multivariable logistic regression model. 
Table 6 | Linear regression analyses of cognitive decline in PD cohort.

\begin{tabular}{llccc}
\hline & & Estimate & SE & $\boldsymbol{p}$-value \\
\hline MMSE score & Intercept & 3.60 & - & - \\
decrease & $1 /$ A $\beta_{42}$ & 1139.92 & 329.08 & 0.012 \\
& MMSE score at & -0.17 & 0.08 & 0.038 \\
& baseline & & & \\
& Follow-up & 0.18 & 0.08 & 0.029 \\
& duration (years) & & & \\
\hline MoCA score & Intercept & 2.75 & - & - \\
decrease & $1 /$ A $\beta_{42}$ & 1395.25 & 482.93 & 0.007 \\
& MoCA score at & -0.08 & 0.09 & 0.387 \\
& baseline & & & \\
& Follow-up & 0.22 & 0.11 & 0.048 \\
& duration (years) & & & \\
\hline
\end{tabular}

A clear positive association was also observed between t-tau and $t-\alpha$-syn in PD group. These findings are consistent with the observation of Kang and coworkers, describing the occurrence of lower CSF levels of t-tau and $t-\alpha$-syn in PD patients. A reasonable explanation may be the mutual interaction of the two molecules, leading to a reduced release of tau in CSF. The discriminative power between PD and OND significantly improved when considering both $\mathrm{o} / \mathrm{t}-\alpha$-syn ratio and $\mathrm{A} \beta_{42} / \mathrm{t}$-tau ratio, as shown by the logistic regression analysis, and further illustrated in Figure 2. This confirms that the combination of several biomarkers is more helpful than single biomarkers for adding diagnostic accuracy of PD.

About the predictive value of CSF biomarkers for cognitive decline in $\mathrm{PD}$, our study confirmed the specific role of low CSF levels of $A \beta_{42}$ in this pathological condition; no other biomarker was significantly associated to this outcome measure. For assessing cognitive function along time, we used both MMSE and MoCA (Gill et al., 2008). Both neuropsychological instruments showed to be related to CSF $A \beta_{42}$ levels, i.e., lower the CSF $\mathrm{A} \beta_{42}$ levels, greater the decrease in MMSE and MoCA scores. The same holds true for $A \beta_{42} / t$-tau ratio with respect to MMSE. Multivariate analysis (adjusting for follow-up time and baseline measurements) confirmed that low CSF $\mathrm{A} \beta_{42}$ levels are independent predictor of cognitive decline in $\mathrm{PD}$, either measured by MMSE or MOCA.

In conclusion, this study further contributes to the evidence of the usefulness of CSF biomarkers for PD diagnosis and prognosis. Major points are the need to combine several CSF biomarkers for improving the diagnostic accuracy, and the confirmed role of low CSF $A \beta_{42}$ levels as independent predictor of cognitive decline in PD. Longitudinal studies measuring biomarkers and clinical parameters over several years represent a major contribution in this field. Analogously to the longitudinal AD Neuroimaging Initiative (ADNI, http://adni-info.org/) in $\mathrm{AD}$, the Parkinson's Progression Markers Initiative (PPMI, http:// ppmi-ifo.org/) will give important knowledge in the field of PD, thanks to the measurement of several CSF, blood, and imaging biomarkers in early de novo PD followed up for several years.

\section{ACKNOWLEDGMENTS}

We would like to thank Mr. Cristiano Spaccatini for his technical assistance. Prof. Lucilla Parnetti and Prof. Omar El-Agnaf received grants from Michael J. Fox Foundation for Parkinson's disease.

\section{REFERENCES}

Aerts, M. B., Esselink, R. A., Abdo, W. F., Bloem, B. R., and Verbeek, M. M. (2012). CSF $\alpha$-synuclein does not differentiate between parkinsonian disorders. Neurobiol. Aging 33, 430.e1-430.e3. doi: 10.1016/j.neurobiolaging.2010. 12.001

Alves, G., Brønnick, K., Aarsland, D., Blennow, K., Zetterberg, H., Ballard, C., et al. (2010). CSF amyloid-beta and tau proteins, and cognitive performance, in early and untreated Parkinson's disease: the Norwegian ParkWest study. J. Neurol. Neurosurg. Psychiatry 81, 1080-1086.doi: 10.1136/jnnp.2009.199950

Balducci, C., Pierguidi, L., Persichetti, E., Parnetti, L., Sbaragli, M., Tassi, C., et al. (2007). Lysosomal hydrolases in cerebrospinal fluid from subjects with Parkinson's disease. Mov. Disord. 22, 1481-1484. doi: 10.1002/mds.21399

Braak, H., Del Tredici, K., Rüb, U., de Vos, R. A., Jansen Steur, E. N., and Braak, E. (2003). Staging of brain pathology related to sporadic Parkinson's disease. Neurobiol. Aging 24, 197-211. doi: 10.1016/S0197-4580(02)00065-9

Ciaccioli, G., Martins, A., Rodrigues, C., Vieira, H., and Calado, P. (2013). A powerful yeast model to investigate the synergistic interaction of $\alpha$-synuclein and tau in neurodegeneration. PLOS ONE 8:e55848. doi: 10.1371/journal.pone.0055848

Compta, Y., Parkkinen, L., O’Sullivan, S. S., Vandrovcova, J., Holton, J. L., Collins, C., et al. (2011). Lewy- and Alzheimer-type pathologies in Parkinson's disease dementia: which is more important? Brain 134(Pt 5), 1493-1505. doi: 10.1093/brain/awr031

Compta, Y., Martí, M. J., Ibarretxe-Bilbao, N., Junqué, C., Valldeoriola, F., Muñoz, E., et al. (2009). Cerebrospinal tau, phospho-tau, and beta-amyloid and neuropsychological functions in Parkinson's disease. Mov. Disord. 24, 2203-2210. doi: $10.1002 / \mathrm{mds} .22594$

Dubois, B., Feldman, H. H., Jacova, C., Cummings, J. L., Dekosky, S. T., BarbergerGateau, P., et al. (2010). Revising the definition of Alzheimer's disease: a new lexicon. Lancet Neurol. 9, 1118-1127. doi: 10.1016/S1474-442270223-442270224

Eusebi, P. (2013). Diagnostic accuracy measures. Cerebrovasc. Dis. 36, 267-272. doi: $10.1159 / 000353863$

Folstein, M. F., Folstein, S. E., and McHugh, P. R. (1975). Mini-mental state. A practical method for grading the cognitive state of patients for the clinician. J. Psychiatr. Res. 12, 189-198. doi: 10.1016/0022-3956(75)90026-6

Gill, D. J., Freshman, A., Blender, J. A., and Ravina, B. (2008). The montreal cognitive assessment as a screening tool for cognitive impairment in Parkinson's disease. Mov. Disord. 23, 1043-1046. doi: 10.1002/mds.22017

Hong, Z., Shi, M., Chung, K. A., Quinn, J. F., Peskind, E. R., Galasko, D., et al. (2010). DJ-1 and alpha-synuclein in human cerebrospinal fluid as biomarkers of Parkinson's disease. Brain 133(Pt 3), 713-726. doi: 10.1093/brain/awq008

Irwin, D. J., White, M. T., Toledo, J. B., Xie, S. X., Robinson, J. L., Van Deerlin, V., et al. (2012). Neuropathologic substrates of Parkinson disease dementia. Ann. Neurol. 72, 587-598. doi: 10.1002/ana.23659

Kang, J. H., Irwin, D. J., Chen-Plotkin, A. S., Siderowf, A., Caspell, C., Coffey, C. S., et al. (2013). Association of cerebrospinal fluid $\beta$-Amyloid 1-42, T-tau, Ptau181, and $\alpha$-synuclein levels with clinical features of drug-naive patients with early Parkinson disease. JAMA Neurol. 70, 1277-1287. doi: 10.1001/jamaneurol. 2013.3861

Leverenz, J. B., Watson, G. S., Shofer, J., Zabetian, C. P., Zhang, J., and Montine, T. J. (2011). Cerebrospinal fluid biomarkers and cognitive performance in nondemented patients with Parkinson's disease. Parkinsonism Relat. Disord. 17, 61-64. doi: 10.1016/j.parkreldis.2010.10.003

Mollenhauer, B., Locascio, J. J., Schulz-Schaeffer, W., Sixel-Döring, F., Trenkwalder, C., and Schlossmacher, M. G. (2011). $\alpha$-Synuclein and tau concentrations in cerebrospinal fluid of patients presenting with parkinsonism: a cohort study. Lancet Neurol. 10, 230-240. doi: 10.1016/S1474-442270014-X

Montine, T. J., Shi, M., Quinn, J. F., Peskind, E. R., Craft, S., Ginghina, C., et al. (2010). CSF A $\beta(42)$ and tau in Parkinson's disease with cognitive impairment. Mov. Disord. 25, 2682-2685. doi: 10.1002/mds.23287

Noguchi-Shinohara, M., Tokuda, T., Yoshita, M., Kasai, T., Ono, K., Nakagawa, M., et al. (2009). CSF alpha-synuclein levels in dementia with Lewy bodies and Alzheimer's disease. Brain Res. 1251, 1-6. doi: 10.1016/j.brainres.2008.11.055 
Paleologou, K. E., Kragh, C. L., Mann, D. M., Salem, S. A., Al-Shami, R., Allsop, D., et al. (2009). Detection of elevated levels of soluble alpha-synuclein oligomers in post-mortem brain extracts from patients with dementia with Lewy bodies. Brain 132(Pt 4)1093-1101. doi: 10.1093/brain/awn349

Park, M. J., Cheon, S. M., Bae, H. R., Kim, S. H., and Kim, J. W. (2011). Elevated levels of $\alpha$-synuclein oligomer in the cerebrospinal fluid of drug-naïve patients with Parkinson's disease. J. Clin. Neurol. 7, 215-222. doi: 10.3988/jcn.2011.7. 4.215

Parnetti, L., Castrioto, A., Chiasserini, D., Persichetti, E., Tambasco, N., El-Agnaf, O., et al. (2013). Cerebrospinal fluid biomarkers in Parkinson disease. Nat. Rev. Neurol. 9, 131-140. doi: 10.1038/nrneurol.2013.10

Parnetti, L., Chiasserini, D., Persichetti, E., Eusebi, P., Varghese, S., Qureshi, M. M., et al. (2014). Cerebrospinal fluid lysosomal enzymes and $\alpha$-synuclein in Parkinson's disease. Mov. Disord. doi: 10.1002/mds.25772. [Epub ahead of print].

Parnetti, L., Chiasserini, D., Bellomo, G., Giannandrea, D., De Carlo, C., Qureshi, M. M., et al. (2011). Cerebrospinal fluid Tau/ $\alpha$-synuclein ratio in Parkinson's disease and degenerative dementias. Mov. Disord. 26, 1428-1435. doi: $10.1002 / \mathrm{mds} .23670$

Parnetti, L., Tiraboschi, P., Lanari, A., Peducci, M., Padiglioni, C., D’Amore, C., et al. (2008). Cerebrospinal fluid biomarkers in Parkinson's disease with dementia and dementia with Lewy bodies. Biol. Psychiatry 64, 850-855. doi: 10.1016/j.biopsych.2008.02.016

Pøikrylová Vranová, H., Mareš, J., Nevrlý, M., Stejskal, D., Zapletalová, J., Hluštík, P., et al. (2010). CSF markers of neurodegeneration in Parkinson's disease. J. Neural. Transm. 117, 1177-1181. doi: 10.1007/s00702-010-0462-z

R Core Team. (2013). R: A. Language and Environment for Statistical Computing. Vienna: R Foundation for Statistical Computing. Available online at: http:// www.R-project.org/

Robin, X., Turck, N., Hainard, A., Tiberti, N., Lisacek, F., Sanchez, J. C., et al. (2011). pROC: an open-source package for $\mathrm{R}$ and $\mathrm{S}+$ to analyze and compare ROC curves. BMC Bioinformatics 12:77. doi: 10.1186/14712105-12-77

Rubin, D. B. (1987). Multiple Imputation for Nonresponse in Surveys. New York, NY; Chichester: Wiley. doi: 10.1002/9780470316696

Siderowf, A., Xie, S. X., Hurtig, H., Weintraub, D., Duda, J., Chen-Plotkin, A., et al. (2010). CSF amyloid \{beta\} 1-42 predicts cognitive decline in Parkinson disease. Neurology 75, 1055-1061. doi: 10.1212/WNL.0b013e3181 f39a78

Sierks, M. R., Chatterjee, G., McGraw, C., Kasturirangan, S., Schulz, P., and Prasad, S. (2011). CSF levels of oligomeric alpha-synuclein and beta-amyloid as biomarkers for neurodegenerative disease. Integr. Biol. (Camb.) 3, 1188-1196. doi: $10.1039 /$ clib00018g

Sjögren, M., Davidsson, P., Wallin, A., Granérus, A. K., Grundström, E., Askmark, H., et al. (2002). Decreased CSF-beta-amyloid 42 in Alzheimer's disease and amyotrophic lateral sclerosis may reflect mismetabolism of beta-amyloid induced by disparate mechanisms. Dement. Geriatr. Cogn. Disord. 13, 112-118. doi: $10.1159 / 000048642$

Spies, P. E., Slats, D., Rikkert, M. G., Tseng, J., Claassen, J. A., and Verbeek, M. M. (2011). CSF $\alpha$-synuclein concentrations do not fluctuate over hours and are not correlated to amyloid $\beta$ in humans. Neurosci. Lett. 504, 336-338. doi: 10.1016/j.neulet.2011.09.063
Spies, P. E., Melis, R. J., Sjögren, M. J., Rikkert, M. G., and Verbeek, M. M. (2009). Cerebrospinal fluid alpha-synuclein does not discriminate between dementia disorders. J. Alzheimers Dis. 16, 363-369. doi: 10.3233/JAD-2009-0955

Tateno, F., Sakakibara, R., Kawai, T., Kishi, M., and Murano, T. (2012). Alpha-synuclein in the cerebrospinal fluid differentiates synucleinopathies (Parkinson Disease, dementia with Lewy bodies, multiple system atrophy) from Alzheimer disease. Alzheimer Dis. Assoc. Disord. 26, 213-216. doi: 10.1097/WAD.0b013e31823899cc

Tokuda, T., Qureshi, M. M., Ardah, M. T., Varghese, S., Shehab, S. A., Kasai, T., et al. (2010). Detection of elevated levels of $\alpha$-synuclein oligomers in CSF from patients with Parkinson disease. Neurology 75, 1766-1772. doi: 10.1212/WNL.0b013e3181fd613b

Tokuda, T., Salem, S. A., Allsop, D., Mizuno, T., Nakagawa, M., Qureshi, M. M., et al. (2006). Decreased alpha-synuclein in cerebrospinal fluid of aged individuals and subjects with Parkinson's disease. Biochem. Biophys. Res. Commun. 349, 162-166. doi: 10.1016/j.bbrc.2006.08.024

Tsigelny, I. F., Crews, L., Desplats, P., Shaked, G. M., Sharikov, Y., Mizuno, H., et al. (2008). Mechanisms of hybrid oligomer formation in the pathogenesis of combined Alzheimer's and Parkinson's diseases. PLoS ONE 3:e3135. doi: 10.1371/journal.pone.0003135

Vekrellis, K., Xilouri, M., Emmanouilidou, E., Rideout, H. J., and Stefanis, L. (2011). Pathological roles of $\alpha$-synuclein in neurological disorders. Lancet Neurol. 10, 1015-1025. doi: 10.1016/S1474-442270213-442270217

Waxman, E. A., and Giasson, B. I. (2011). Induction of intracellular tau aggregation is promoted by $\alpha$-synuclein seeds and provides novel insights into the hyperphosphorylation of tau. J. Neurosci. 31, 7604-7618. doi: 10.1523/JNEUROSCI.0297-0211.2011

Zhang, J., Mattison, H. A., Liu, C., Ginghina, C., Auinger, P., McDermott, M. P., et al. (2013). Longitudinal assessment of tau and amyloid beta in cerebrospinal fluid of Parkinson disease. Acta Neuropathol. 126, 671-682. doi: 10.1007/s00401-013-1121-x

Zhang, J., Sokal, I., Peskind, E. R., Quinn, J. F., Jankovic, J., Kenney, C., et al. (2008). CSF multianalyte profile distinguishes Alzheimer and Parkinson diseases. Am. J. Clin. Pathol. 129, 526-529. doi: 10.1309/W01Y0B808EMEH12L

Conflict of Interest Statement: The authors declare that the research was conducted in the absence of any commercial or financial relationships that could be construed as a potential conflict of interest.

Received: 15 January 2014; accepted: 05 March 2014; published online: 31 March 2014. Citation: Parnetti L, Farotti L, Eusebi P, Chiasserini D, De Carlo C, Giannandrea D, Salvadori N, Lisetti V, Tambasco N, Rossi A, Majbour NK, El-Agnaf O, and Calabresi $P$ (2014) Differential role of CSF alpha-synuclein species, tau, and A $\beta 42$ in Parkinson's Disease. Front. Aging Neurosci. 6:53. doi: 10.3389/fnagi.2014.00053

This article was submitted to the journal Frontiers in Aging Neuroscience.

Copyright (C) 2014 Parnetti, Farotti, Eusebi, Chiasserini, De Carlo, Giannandrea, Salvadori, Lisetti, Tambasco, Rossi, Majbour, El-Agnaf, and Calabresi. This is an openaccess article distributed under the terms of the Creative Commons Attribution License (CC BY). The use, distribution or reproduction in other forums is permitted, provided the original author(s) or licensor are credited and that the original publication in this journal is cited, in accordance with accepted academic practice. No use, distribution or reproduction is permitted which does not comply with these terms. 